\begin{tabular}{|c|c|}
\hline Title & Interdot spin transfer dynamics in laterally coupled excited spin ensemble of high-density InGaA s quantum dots \\
\hline Author(s) & $\begin{array}{l}\text { Hiura, Satoshi; Takeishi, Kazuki; U rabe, Masay uki; Itabashi, Kodai; Takay ama, Junichi; Kiba, Takayuki; Sueoka, } \\
\text { Kazuhisa; Muray ama, A kihiro }\end{array}$ \\
\hline Citation & $\begin{array}{l}\text { A pplied phy sics letters, 113(2), } 023104 \\
\text { https://doi.org/10.1063/1.5022641 }\end{array}$ \\
\hline Issue Date & 2018-07-09 \\
\hline Doc URL & http:/hdl.handle.net/2115/74910 \\
\hline Rights & $\begin{array}{l}\text { This article may be downloaded for personal use only. A ny other use requires prior permission of the author and AIP } \\
\text { Publishing.T he following article appeared in A ppl. Phys. Lett. 113(2), 023104 (2018) and may be found at } \\
\text { https://doi.org/10.1063/1.5022641. }\end{array}$ \\
\hline Type & article \\
\hline File Information & 1.5022641.pdf \\
\hline
\end{tabular}

Instructions for use 


\section{Interdot spin transfer dynamics in laterally coupled excited spin ensemble of high- density InGaAs quantum dots}

Satoshi Hiura, Kazuki Takeishi, Masayuki Urabe, Kodai Itabashi, Junichi Takayama, Takayuki Kiba, Kazuhisa Sueoka, and Akihiro Murayama

Citation: Appl. Phys. Lett. 113, 023104 (2018); doi: 10.1063/1.5022641

View online: https://doi.org/10.1063/1.5022641

View Table of Contents: http://aip.scitation.org/toc/apl/113/2

Published by the American Institute of Physics

\section{Articles you may be interested in}

Resonance fluorescence from an atomic-quantum-memory compatible single photon source based on GaAs droplet quantum dots

Applied Physics Letters 113, 021102 (2018); 10.1063/1.5034402

Mid-wavelength high operating temperature barrier infrared detector and focal plane array

Applied Physics Letters 113, 021101 (2018); 10.1063/1.5033338

Automated tuning of inter-dot tunnel coupling in double quantum dots

Applied Physics Letters 113, 033101 (2018); 10.1063/1.5031034

Strongly temperature-dependent recombination kinetics of a negatively charged exciton in asymmetric quantum dots at $1.55 \mu \mathrm{m}$

Applied Physics Letters 113, 043103 (2018); 10.1063/1.5030895

Independent indistinguishable quantum light sources on a reconfigurable photonic integrated circuit Applied Physics Letters 112, 211104 (2018); 10.1063/1.5028339

Electrical properties of extended defects in strain relaxed GeSn

Applied Physics Letters 113, 022102 (2018); 10.1063/1.5034573

\section{Conference Proceedings}




\title{
Interdot spin transfer dynamics in laterally coupled excited spin ensemble of high-density InGaAs quantum dots
}

\author{
Satoshi Hiura, ${ }^{1, a)}$ Kazuki Takeishi, ${ }^{1}$ Masayuki Urabe, ${ }^{1}$ Kodai Itabashi, ${ }^{1}$ Junichi Takayama, ${ }^{1}$ \\ Takayuki Kiba, $^{2}$ Kazuhisa Sueoka, ${ }^{1}$ and Akihiro Murayama ${ }^{1}$ \\ ${ }^{1}$ Graduate School of Information Science and Technology, Hokkaido University, Kita 14, Nishi 9, Kita-ku, \\ Sapporo 060-0814, Japan \\ ${ }^{2}$ Kitami Institute of Technology, 165 Koen-cho, Kitami 090-8507, Japan
}

(Received 17 January 2018; accepted 30 June 2018; published online 12 July 2018)

\begin{abstract}
Interdot spin transfer dynamics is studied in a laterally coupled excited spin ensemble of highdensity InGaAs quantum dots (QDs). We observe a rise time of the photoluminescence intensity of $\sim 100$ ps and a simultaneous increase in the spin polarization of the excited spin ensemble, indicating spin injection from higher-energy levels in smaller QDs. Moreover, this coupled ensemble exhibits decay properties of the spin polarization that vary with the excited spin density. This phenomenon can be quantitatively understood by considering interdot spin transfer into lower-energy levels of the surrounding QDs, where the transfer rate depends on the degree of state filling of each QD level. Published by AIP Publishing. https://doi.org/10.1063/1.5022641
\end{abstract}

Carrier dynamics of quantum dot (QD) structures has attracted much attention due to its potential applications in optoelectronic devices such as wavelength switching devices ${ }^{1}$ and semiconductor lasers. ${ }^{2}$ More recently, spin dynamics in self-assembled QDs of III-V compound semiconductors generated significant interest due to the long spin lifetime of the associated carriers or excitons. ${ }^{3-6}$ This suppression of spin relaxation has led to extensive studies of spin injection and relaxation dynamics in $\mathrm{InGaAs}_{\mathrm{QDs}}{ }^{7,8}$ and fabrication of optospintronic devices such as spin-polarized light-emitting diodes $^{9-11}$ or laser diodes ${ }^{12}$ that use QDs as active layers. High-density QD systems are essential for future QD-based optospintronic devices as they can provide sufficiently high optical gains in laser structures. An important phenomenon in high-density QDs is the interdot carrier and spin transfer, which includes interdot tunneling and subsequent intradot relaxation processes and can be affected by the characteristic for QDs state filling effect. ${ }^{13}$ Of particular importance for the QD-based spintronic device development is the investigation of spin transfer dynamics in coupled excited spin ensembles of high-density QDs.

In this work, circularly polarized photoluminescence (PL) spectroscopy, time-resolved PL (TRPL) measurements including a rate-equation analysis that takes into account interdot transfer, and three-dimensional calculation of the electron and hole wavefunctions are performed to study the interdot spin transfer inevitably present in high-density QDs. Laterally coupled high-density InGaAs QDs are grown by utilizing a misoriented $\mathrm{GaAs}(100)$ substrate. ${ }^{14} \mathrm{We}$ mainly discuss the transient behavior of the circular polarization of the PL in QDs, which reflects the time-dependent spin polarization of the excited states in the QD ensemble. From the rise times of the PL and the corresponding circular polarization degree (CPD), we obtain the optical spin injection dynamics in the laterally coupled excited spin ensemble; we further discuss the interdot relaxation dynamics of electron spins based on the decay properties of the PL and CPD.

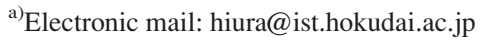

Here, the CPD is defined as $\left(I_{\sigma+}-I_{\sigma-}\right) /\left(I_{\sigma+}+I_{\sigma-}\right)$, where $I_{\sigma+}$ and $I_{\sigma-}$ denote the intensities of the $\sigma^{+}$- and $\sigma^{-}$-polarized PL signals, respectively.

Laterally uncoupled and coupled QDs were grown on misoriented GaAs(100) substrates with GaAs buffer layers by molecular beam epitaxy under growth conditions similar to those used previously. ${ }^{7,8}$ A single layer of self-assembled $\mathrm{In}_{0.5} \mathrm{Ga}_{0.5} \mathrm{As} \mathrm{QDs}$ with $6 \mathrm{ML}$ was grown at growth rates of 0.10 and $0.20 \mathrm{ML} / \mathrm{s}$, and subsequently capped with $40 \mathrm{~nm}$ thick GaAs layer. Additional QDs were grown on this capping layer to investigate their structures by atomic force microscopy (AFM). The separation of $40 \mathrm{~nm}$ is long enough to suppress the interdot tunneling of carriers along the vertical direction, and emissions from the surface QDs should appear in a significantly lower energy region. ${ }^{7,15}$ Circularly polarized TRPL curves were obtained at $6 \mathrm{~K}$ using a method described previously. ${ }^{7,8}$ Furthermore, to investigate the lateral coupling strength between the electron wavefunctions of QDs, three-dimensional calculations of the electronic band structure and the wavefunctions of carriers in two QD samples with different interdot distances were carried out using the nextnano ${ }^{3}$ software package, ${ }^{16}$ taking into account the size and shape of the dots, their composition, and strain, as well as the non-uniform distribution of In atoms in $\mathrm{In}_{0.5} \mathrm{Ga}_{0.5}$ As QDs. ${ }^{17,18}$

Figures 1(a) and 1(b) show the AFM images of the QDs grown at growth rates of 0.10 and $0.20 \mathrm{ML} / \mathrm{s}$, respectively. The QDs are well ordered along the [011] direction in a onedimensional chain structure, which is similar to previously reported InGaAs QDs grown on a misoriented GaAs substrate. ${ }^{14}$ The lateral densities of the QDs can be estimated as $3.5 \times 10^{10}$ and $8.1 \times 10^{10} \mathrm{~cm}^{-2}$. The averaged distances between QD centers are $\sim 60$ and $\sim 30 \mathrm{~nm}$ (hereinafter denoted as $\mathrm{D}=60$ and $30 \mathrm{~nm}$ ), as indicated by the cross-sectional line profiles in Figs. 1(c) and 1(d). Here, considering the average QD size of $\sim 20 \mathrm{~nm}$ obtained by AFM analysis, the base-tobase distances of the QDs are 40 and $10 \mathrm{~nm}$ for $\mathrm{D}=60$ and $30 \mathrm{~nm}$, respectively. These QD height profiles also show the inhomogeneous height distribution of the QD sample grown at a faster growth rate, which corresponds to the inhomogeneous 

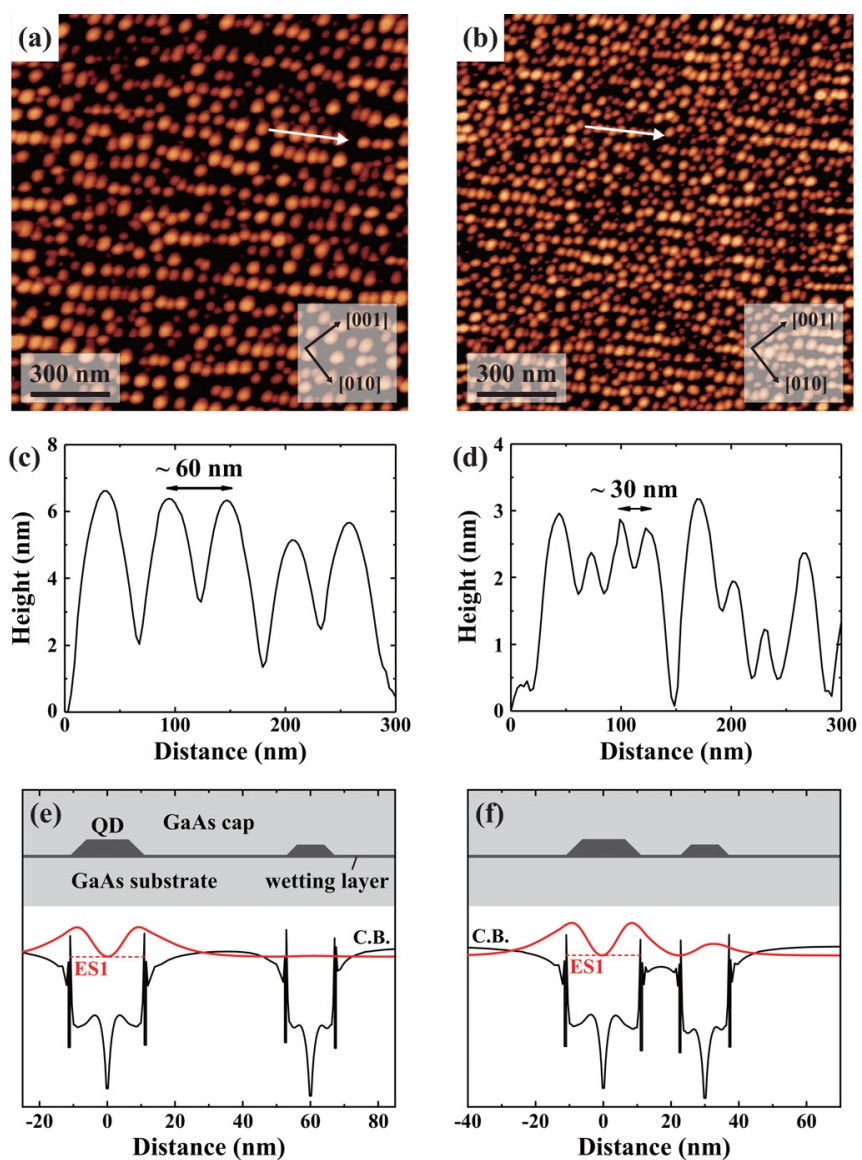

FIG. 1. (a) and (b) AFM images of $\operatorname{In}_{0.5} \mathrm{Ga}_{0.5} \mathrm{As}$ QDs grown at growth rates of 0.10 and $0.20 \mathrm{ML} / \mathrm{s}$, respectively. (c) and (d) Cross-sectional line profiles taken along the white lines in (a) and (b), respectively. (e) and (f) Conduction band profiles (C.B.) calculated for two QD systems with the center-to-center distances of 60 and $30 \mathrm{~nm}$, respectively. The base lengths of two QDs are set to 24 and $16 \mathrm{~nm}$, whose values are determined from the QD sizes obtained by AFM analysis. The solid red curves show the distribution of the existence probabilities of electron wavefunctions at the first excited state (ES1).

QD size distribution assuming a constant aspect ratio of QDs. ${ }^{19}$ Figures 1(e) and 1(f) show typical results of three-dimensional calculations of conduction band profiles and first excited state (ES1) including the distribution of the existence probabilities of electron wavefunctions along the lateral direction in two QD samples with $\mathrm{D}=60$ and $30 \mathrm{~nm}$, respectively. Here, the base lengths of two lateral QDs were set to 24 and $16 \mathrm{~nm}$. These values are determined from the QD sizes obtained by AFM analysis. As seen in Figs. 1(e) and 1(f), the central parts of the QDs are associated with deeper conduction band edges owing to the correspondingly higher In content. ${ }^{17,18}$ These calculation results clearly show that the electron wavefunction for $D=60 \mathrm{~nm}$ is strongly localized at larger QDs, while the degree of wavefunction penetration into smaller QDs becomes more significant for $\mathrm{D}=30 \mathrm{~nm}$. This implies significantly higher probabilities of electron tunneling from smaller to larger QDs for $\mathrm{D}=30 \mathrm{~nm}$ through the wavefunction coupling (tunneling) and the subsequent intradot relaxation. Hereinafter, the QD samples with $\mathrm{D}=60$ and $30 \mathrm{~nm}$ are defined as laterally uncoupled and coupled QD ensembles, respectively.

Circularly polarized time-integrated PL spectra for the two QD samples measured at an excitation power of $2.5 \mathrm{~mW}$ and the corresponding CPD as a function of photon energy are shown in Figs. 2(a) and 2(b). The broad PL spectra
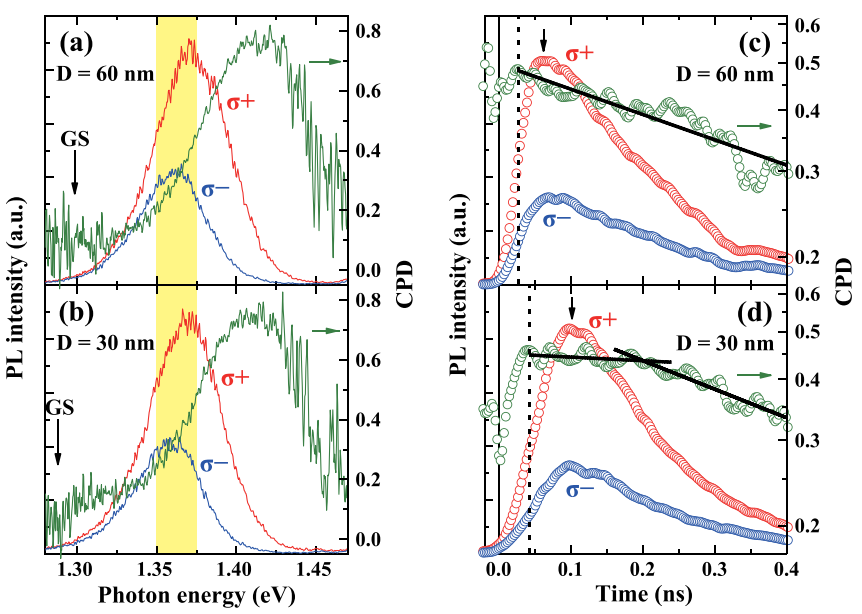

FIG. 2. (a) and (b) Circularly polarized time-integrated PL spectra; (c) and (d) TRPL curves and corresponding CPD for ES1 [indicated by the yellow rectangles in (a) and (b)] for the QD samples with $\mathrm{D}=60$ and $30 \mathrm{~nm}$. The ground state (GS) is indicated by the black arrows in (a) and (b). The black solid lines indicate the fit results for single exponential decay curves of time-dependent CPD.

peaked at $\sim 1.36-1.37 \mathrm{eV}$ are mainly originated from the ES1 of excitons in QDs. At lower energies, the obtained spectral shapes are affected by the detection limit of our streak camera. ${ }^{7}$ For reference, the ground states (GSs) of the two samples, detected with a charge-coupled device, are indicated by the black arrows in Figs. 2(a) and 2(b). Both QD samples show similar CPD spectra, with slightly higher CPD values observed for the coupled QD ensembles. Figures 2(c) and 2(d) show the circularly polarized TRPL curves and the corresponding CPD for the same samples, obtained for the range of photon energies indicated by the yellow rectangles in Figs. 2(a) and 2(b). Three main differences can be observed when comparing these TRPL curves. First, the PL rise times, indicated by the black arrows, are different. The much longer PL rise time of $\sim 100$ ps for the coupled QD ensemble reflects the lateral electronic coupling of QDs and the coexistence of smaller and larger QDs. This PL behavior is due to the delayed carrier filling of larger QDs, which provide a dominant contribution to the PL emission, by smaller QDs attributed to carrier transfer from smaller to larger QDs. ${ }^{20-24}$ Second, the characteristic times of the initial CPD increase, indicated by the dashed black lines, are different. The longer CPD increase observed for the coupled QD ensemble can be closely related to the slower PL rise discussed above. The GS in the laterally coupled QD ensemble should be occupied more slowly due to the interdot transfer. This slower filling of the GS can lead to the longer initial increase in the CPD for ES1 due to the blocked relaxation of the majority-spin electrons from the ES1. ${ }^{25}$ For the minorityspin electrons, the relaxation is possible, which defines the transient CPD behavior immediately after its initial increase. The CPD for the coupled excited spin ensemble persists for $\sim 0.15 \mathrm{~ns}$, as indicated by the black solid line showing the first slower component of CPD decay, and then decreases with a faster CPD decay time. In contrast, the CPD for the uncoupled one is decayed with only a single exponential decay, according to the inherent spin relaxation in QDs. ${ }^{6-8}$ In the following, only the laterally coupled QD sample is 
discussed, because this coupled ensemble shows the unique transient behavior of the CPD, as compared to the wellknown spin relaxation dynamics in the laterally uncoupled QD sample.

The above described AFM and TRPL results indicate that the laterally coupled QD ensemble consists of smaller and larger QDs, and that each interdot distance is sufficiently small to allow interdot spin transfer among QDs. To analyze the circularly polarized TRPL curves for the coupled QD ensemble, we use a rate-equation model including an interdot spin transfer parameter, which was not taken into account in the previously reported rate-equation model. ${ }^{7,8} \mathrm{In}$ our model, three types of QD levels, including the higherenergy level in smaller QD (SQD), the mainly focused level in larger QD1, and the lower-energy sub-level in neighboring QD2, are taken into account. Here, note that these three levels belong to different QDs coupled to each other. In this model, spin injection process from the barrier into the QD1 and QD2 levels via the SQD level is assumed owing to the faster capture rate of carriers from the barrier into the SQDs. ${ }^{26}$ The model is schematically illustrated in Fig. 3(a). The rate equations for the laterally coupled excited spin ensemble can be written as follows:

$$
\begin{aligned}
\frac{d N_{0}}{d t}= & \frac{-N_{0}}{\tau_{\mathrm{inj}}}\left[\eta^{+}\left(1-\frac{N_{\mathrm{SQ}}^{\sigma+}}{D_{\mathrm{SQ}}}\right)+\eta^{-}\left(1-\frac{N_{\mathrm{SQ}}^{\sigma-}}{D_{\mathrm{SQ}}}\right)\right], \\
\frac{d N_{\mathrm{SQ}}^{\sigma \pm}}{d t}= & \frac{N_{0}}{\tau_{\mathrm{inj}}} \eta^{ \pm}\left(1-\frac{N_{\mathrm{SQ}}^{\sigma \pm}}{D_{\mathrm{SQ}}}\right)-\frac{N_{\mathrm{SQ}}^{\sigma \pm}}{\tau_{\mathrm{r}}} \mp \frac{N_{\mathrm{SQ}}^{\sigma+}}{\tau_{\mathrm{s}}} \pm \frac{N_{\mathrm{SQ}}^{\sigma-}}{\tau_{\mathrm{S}}} \\
& -\frac{N_{\mathrm{SQ}}^{\sigma \pm}}{\tau_{\mathrm{tr} 1}^{e f}}\left(1-\frac{N_{\mathrm{QD} 1}^{\sigma \pm}}{D_{\mathrm{QD} 1}}\right)-\frac{N_{\mathrm{SQ}}^{\sigma \pm}}{\tau_{\mathrm{tr} 1}^{e f}}\left(1-\frac{N_{\mathrm{QD} 2}^{\sigma \pm}}{D_{\mathrm{QD} 2}}\right), \\
\frac{d N_{\mathrm{QD} 1(2)}^{\sigma \pm}}{d t}= & -\frac{N_{\mathrm{QD} 1(2)}^{\sigma \pm} \mp \frac{N_{\mathrm{QD} 1(2)}^{\sigma+}}{\tau_{\mathrm{r}}} \pm \frac{N_{\mathrm{QD} 1(2)}^{\sigma-}}{\tau_{\mathrm{S}}}+\frac{N_{\mathrm{SQ}}^{\sigma \pm}}{\tau_{\mathrm{tr} 1}^{e f f}}}{} \\
& \times\left(1-\frac{N_{\mathrm{QD} 1(2)}^{\sigma \pm}}{D_{\mathrm{QD} 1(2)}}\right)-(+) \frac{N_{\mathrm{QD} 1}^{\sigma \pm}}{\tau_{\mathrm{tr} 2}^{e f f}}\left(1-\frac{N_{\mathrm{QD} 2}^{\sigma \pm}}{D_{\mathrm{QD} 2}}\right),
\end{aligned}
$$
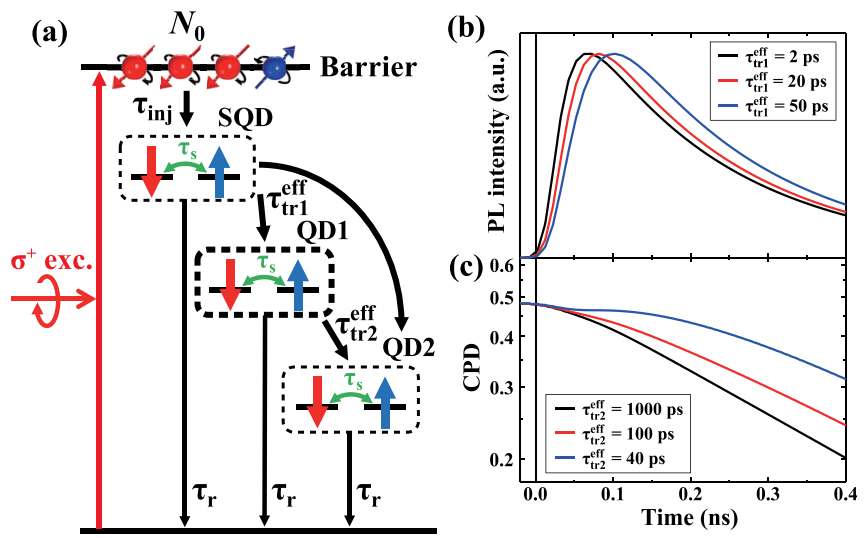

FIG. 3. (a) Rate-equation model including the higher-energy level in smaller QD (SQD), mainly focused level in larger QD1, and lower-energy sub-level in neighboring QD2. Interdot transfer parameters $\tau_{\mathrm{tr} 1}^{e f f}$ and $\tau_{\mathrm{tr} 2}^{e f f}$ are taken into account, which is a key difference with the previous rate-equation model. ${ }^{7,8}$ Rate-equation simulations for the time-resolved (b) PL with various $\tau_{\text {tr1 }}^{\text {eff }}$ and (c) CPD with various $\tau_{\text {tr } 2}^{\text {eff }}$ for QD1. where $N_{0}$ is the initial number of excitons per unit area with the $\sigma^{+}$-polarization. $\eta^{+(-)}$is a fractional parameter reflecting the spin conservation (flip) during the spin-injection process, i.e., $\eta^{+}+\eta^{-}=1$. $\tau_{\text {inj }}, \tau_{\mathrm{r}}$, and $\tau_{\mathrm{s}}$ are time constants for the spin injection from the barrier to the higher-energy SQD level, energy relaxation from three QD levels, and spin relaxation between these spin-polarized levels, respectively. $N_{\mathrm{SQ}}^{\sigma \pm}, N_{\mathrm{QD} 1}^{\sigma \pm}$, and $N_{\mathrm{QD} 2}^{\sigma \pm}$ represent the number of excitons per unit area with $\sigma^{ \pm}$-polarization in the higher-energy SQD, QD1, and lower-energy QD2 levels, respectively. Here, $D_{\mathrm{SQ}}$ and $D_{\mathrm{QD} 1(2)}$ represent the densities of states for these QD levels, i.e., number of states per unit area. The factors $\left(1-N_{\mathrm{SQ}}^{\sigma \pm} / D_{\mathrm{SQ}}\right)$ and $\left(1-N_{\mathrm{QD} 1(2)}^{\sigma \pm} / D_{\mathrm{QD1}(2)}\right)$ are state filling factors for each QD level, indicating that the exciton can be injected into unoccupied QD levels only. ${ }^{13}$ The newly introduced parameters $\tau_{\mathrm{tr} 1}^{e f f}$ and $\tau_{\mathrm{tr} 2}^{\text {eff }}$ mean effective time constants of interdot spin transfer from SQD into QD1(2) and from QD1 into QD2, respectively. Here, intradot relaxation time inside a QD is not directly taken into account, as this ultra-fast relaxation process mainly contributes to the initial CPD increasing feature with a relaxation time shorter than 1 ps. ${ }^{25}$ In this rate-equation fitting, the following CPD decaying features are mainly focused on. The results of rateequation simulations for the TRPL with various $\tau_{\text {trl }}^{\text {eff }}$ and for the time-resolved CPD with various $\tau_{\mathrm{tr} 2}^{\text {eff }}$ for QD1, combined with the results of convolution calculations for the time response of the measurement system, are shown in Figs. 3(b) and 3(c), respectively. As clearly seen in Fig. 3(b), the slower PL rise can be reproduced using a longer $\tau_{\mathrm{tr} 1}^{\text {eff }}$, while in the case of $\tau_{\mathrm{tr} 1}^{e f f}=2 \mathrm{ps}$, which is close to the case without SQD, the slow PL rise cannot be reproduced. This result clearly indicates spin injection from the barrier into the QD1 level via the higher-energy SQD level in the laterally coupled QDs. From the results for the time-resolved $\mathrm{CPD}$, the transient CPD behavior is found to depend on the $\tau_{\mathrm{tr} 2}^{\text {eff }}$ values. Figure 3(c) shows the slower CPD decay with shorter $\tau_{\mathrm{tr} 2}^{\text {eff }}$, in good agreement with the time-resolved CPD results shown in Fig. 2(d).

From the fitting analysis using the above rate-equation model, we can quantitatively separate the individual factors responsible for the transient behavior of the PL and CPD. The circularly polarized TRPL curves and the corresponding CPD measured at excitation powers of $1.0,2.5$, and $7.5 \mathrm{~mW}$ for the laterally coupled QDs, obtained for the range of photon energies indicated by the yellow rectangle in Fig. 2(b), are shown in Figs. 4(a)-4(c). Solid lines show the best-fit calculations of the rate equations. The initial rise of the CPD is slower at the lower excitation power of $1.0 \mathrm{~mW}$ due to the slower filling of the lower-lying states, as discussed earlier; after reaching the maximum, the CPD gradually decreases according to the inherent $\tau_{\mathrm{s}}$ of $\sim 800 \mathrm{ps} .{ }^{6-8}$ When increasing the excitation power to $2.5 \mathrm{~mW}$, the CPD rise time is reduced, which is attributed to the faster filling of the lower states; after reaching the maximum, the CPD remains relatively constant. This unique CPD behavior can be well fit by tuning the two parameters $\tau_{\mathrm{tr} 2}^{\text {eff }}$ and $D_{\mathrm{QD} 2}$, as discussed below. The high state filling strength for the QD level can be observed at the higher excitation power of $7.5 \mathrm{~mW}$.

Figures 5(a) and 5(b) show the excitation power dependences of the interdot transfer time constants, $\tau_{\text {trl,tr2 }}^{\text {eff }}$, and the 


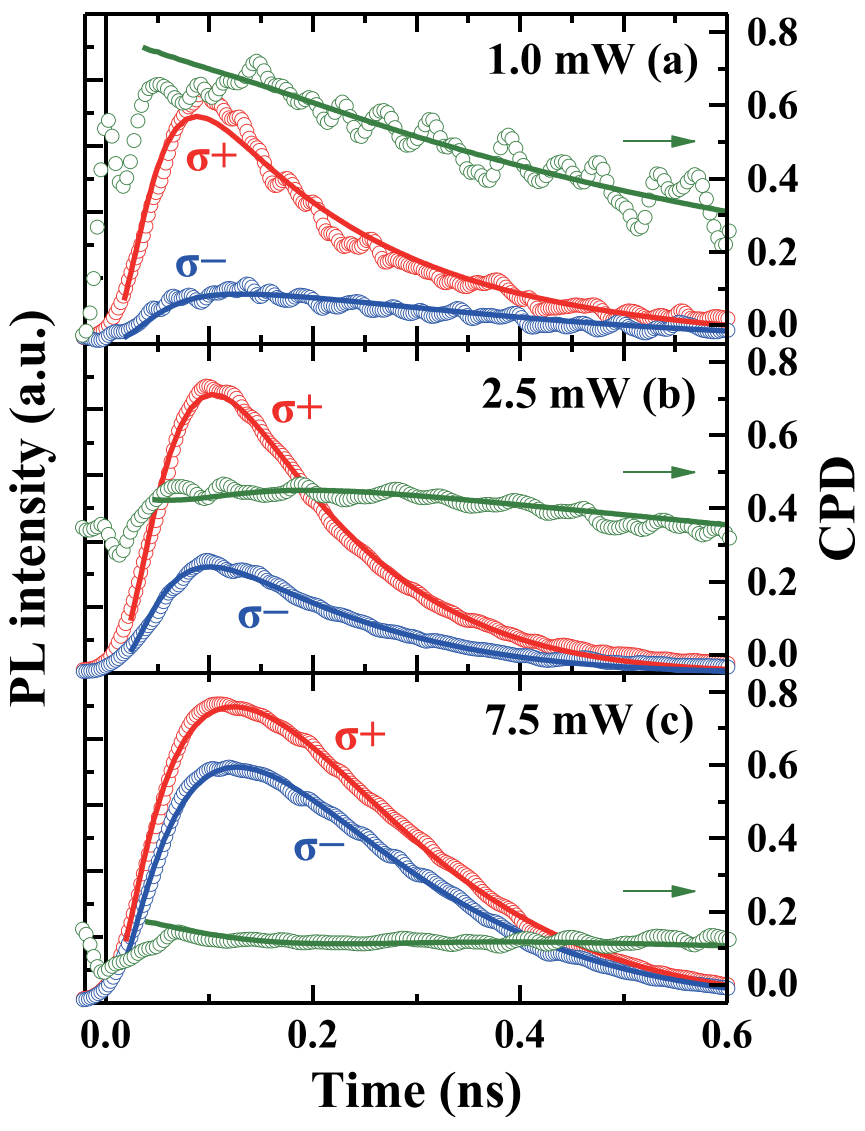

FIG. 4. Circularly polarized TRPL curves and the corresponding CPD measured at excitation powers of (a) 1.0, (b) 2.5 , and (c) $7.5 \mathrm{~mW}$ for the QD sample with $\mathrm{D}=30 \mathrm{~nm}$, best-fitted with rate-equation calculations (solid lines).

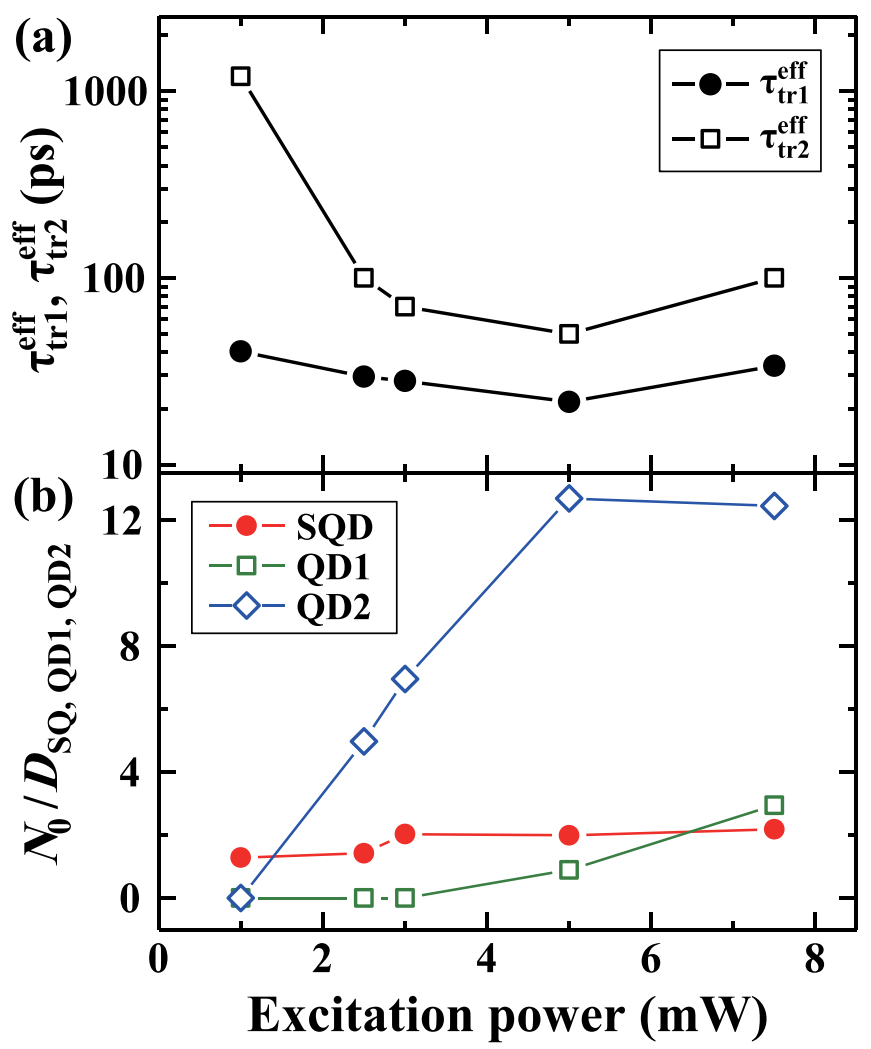

FIG. 5. Excitation power dependence of (a) interdot transfer time constant $\tau_{\text {tr1,tr2 }}^{\text {eff }}$ and (b) degree of state filling of each QD level $N_{0} / D_{\mathrm{SQ}, \mathrm{QD} 1, \mathrm{QD} 2}$, deduced from the rate-equation fit for the QD sample with $\mathrm{D}=30 \mathrm{~nm}$. degree of state filling for each QD level, $N_{0} / D_{\mathrm{SQ}}, \mathrm{QD} 1, \mathrm{QD} 2$, deduced from the above rate-equation fit, respectively. The $\tau_{\mathrm{tr} 1}^{\text {eff }}$ is almost independent of excitation power and is much shorter than $\tau_{\mathrm{tr} 2}^{e f f}$, which is due to the higher energy level for the interdot transfer. For the lowest excitation power of 1 $\mathrm{mW}$, a long $\tau_{\mathrm{tr} 2}^{\text {eff }}$ on the order of $1 \mathrm{~ns}$ is observed. This long $\tau_{\mathrm{tr} 2}^{e f f}$, corresponding to a slow rate of interdot transfer, means that intradot rather than interdot relaxation should be dominant due to the low state filling strength of the lower states. In this case, the highest state filling of the SQD level is observed in Fig. 5(b), which can be ascribed to the limited density of the states due to the small population of the corresponding level in QDs. It should be noted that a much shorter $\tau_{\mathrm{tr} 2}^{\text {eff }}$ of $\sim 100 \mathrm{ps}$ is observed at the higher excitation power of $2.5 \mathrm{~mW}$, where the CPD exhibits a persistent behavior, as shown in Fig. 4(b). This drastically shortened $\tau_{\mathrm{tr} 2}^{\text {eff }}$ demonstrates that the much slower CPD decay compared to the spin relaxation in QDs can be closely related to the faster transfer rate, i.e., interdot relaxation. In this case, the degree of state filling of the QD2 level is also drastically increased, though that of the QD1 level remains low. This higher state filling of the QD2 level suggests the selective transfer of minority-spin electrons from the QD1 into the QD2 level, which is in contrast to the Pauli blocking of the transfer of majority-spin electrons. ${ }^{25}$ As a result, the CPD decay associated with the QD1 level should become suppressed due to the spin redistribution in the coupled QD system. For the higher excitation power of $5 \mathrm{~mW}$, the degree of state filling of the QD1 level as well as that of the QD2 level is increased. The $\tau_{\mathrm{tr} 2}^{\text {eff }}$ becomes gradually shortened with the increase in excitation power up to $5 \mathrm{~mW}$, which indicates that interdot transfer from the QD1 into the QD2 is actively induced by the increase in state filling of the lower-energy level inside the QD1 contributing to the blocking of intradot relaxation. For the highest excitation power of $7.5 \mathrm{~mW}$, the degree of state filling of the QD1 level is largely increased, resulting in the much lower initial CPD. ${ }^{7,8}$ In this case, $\tau_{\mathrm{tr} 2}^{\text {eff }}$ increases with the increase in the excitation power, as state filling of the QD2 level becomes more significant, which can prevent the interdot spin transfer from the QD1 into the QD2 level. Here, note that from Fig. 4(c), the CPD persists for $\sim 0.6 \mathrm{~ns}$ even in the case of the strong state filling. This longterm suppression of the CPD decay might also result from the interdot spin transfer, which is somewhat fast even at the higher excitation power of $7.5 \mathrm{~mW}$ owing to the strong lateral coupling of electron wavefunctions between QDs, as illustrated in Fig. 1(f). These results demonstrate that the transient properties of spin polarization in the laterally coupled excited spin ensemble depend on both the geometrical parameters (size distribution and interdot distance of QDs) and the degree of state filling of each QD level, corresponding to the excited spin density. These findings will provide significant insight for further investigation and development of spin functionalities of high-density InGaAs QD systems.

In summary, interdot spin transfer dynamics in a laterally coupled excited spin ensemble of high-density InGaAs QDs was studied. The slower PL rise and corresponding longer CPD increase from the coupled excited spin ensemble indicate spin injection from the barrier into the main QD level via the higher-energy level of smaller QDs. In addition, 
this coupled spin ensemble demonstrates the dependence of the spin polarization decay properties on the excited spin density. The observed behavior can be quantitatively explained by interdot spin transfer into lower-energy QD levels, with the transfer rate depending on the state filling factor of each QD level.

This study was supported by the Japan Society for the Promotion of Science, Grant-in-Aid for Scientific Research (S) (KAKENHI No. 16H06359), Grant-in-Aid for Research Activity start-up (KAKENHI No. 17H06482), and bilateral program.

${ }^{1}$ W. Zhou, O. Qasaimeh, J. Phillips, S. Krishna, and P. Bhattacharyaa, Appl. Phys. Lett. 74, 783 (1999).

${ }^{2}$ Y. Arakawa and H. Sakaki, Appl. Phys. Lett. 40, 939 (1982).

${ }^{3}$ A. V. Khaetskii and Y. V. Nazarov, Phys. Rev. B 61, 12639 (2000).

${ }^{4}$ M. Paillard, X. Marie, P. Renucci, T. Amand, A. Jbeli, and J. M. Gérard, Phys. Rev. Lett. 86, 1634 (2001).

${ }^{5}$ P. Borri, W. Langbein, S. Schneider, and U. Woggon, Phys. Rev. Lett. 87, 157401 (2001).

${ }^{6}$ A. Tackeuchi, R. Ohtsubo, K. Yamaguchi, M. Murayama, T. Kitamura, T. Kuroda, and T. Takagahara, Appl. Phys. Lett. 84, 3576 (2004).

${ }^{7}$ T. Kiba, X. J. Yang, T. Yamamura, Y. Kuno, A. Subagyo, K. Sueoka, and A. Murayama, Appl. Phys. Lett. 103, 082405 (2013).

${ }^{8}$ T. Yamamura, T. Kiba, X. J. Yang, J. Takayama, A. Subagyo, K. Sueoka, and A. Murayama, J. Appl. Phys. 116, 094309 (2014).

${ }^{9}$ Y. Chye, M. E. White, E. Johnston-Halperin, B. D. Gerardot, D. D. Awschalom, and P. M. Petroff, Phys. Rev. B 66, 201301 (2002).

${ }^{10}$ C. H. Li, G. Kioseoglou, O. M. J. van't Erve, M. E. Ware, D. Gammon, R. M. Stroud, B. T. Jonker, R. Mallory, M. Yasar, and A. Petrou, Appl. Phys. Lett. 86, 132503 (2005).
${ }^{11}$ L. Lombez, P. Renucci, P. F. Braun, H. Carrère, X. Marie, T. Amand, B. Urbaszek, J. L. Gauffier, P. Gallo, T. Camps, A. Arnoult, C. Fontaine, C. Deranlot, R. Mattana, H. Jaffrès, J.-M. George, and P. H. Binh, Appl. Phys. Lett. 90, 081111 (2007).

${ }^{12}$ D. Basu, D. Saha, C. C. Wu, M. Holub, Z. Mi, and P. Bhattacharya, Appl. Phys. Lett. 92, 091119 (2008).

${ }^{13}$ S. Grosse, J. H. H. Sandmann, G. von Plessen, J. Feldmann, H. Lipsanen, M. Sopanen, J. Tulkki, and J. Ahopelto, Phys. Rev. B 55, 4473 (1997).

${ }^{14}$ M. Kitamura, M. Nishioka, J. Oshinowo, and Y. Arakawa, Appl. Phys. Lett. 66, 3663 (1995).

${ }^{15}$ Z. L. Miao, Y. W. Zhang, S. J. Chua, Y. H. Chye, P. Chen, and S. Tripathy, Appl. Phys. Lett. 86, 031914 (2005).

${ }^{16}$ S. Birner, T. Zibold, T. Andlauer, T. Kubis, M. Sabathil, A. Trellakis, and P. Vogl, IEEE Trans. Electron Devices 54, 2137 (2007).

${ }^{17}$ N. Liu, J. Tersoff, O. Baklenov, A. L. Holmes, Jr., and C. K. Shih, Phys. Rev. Lett. 84, 334 (2000).

${ }^{18}$ A. Lenz, R. Timm, H. Eisele, C. Hennig, S. K. Becker, R. L. Sellin, U. W. Pohl, D. Bimberg, and M. Dähne, Appl. Phys. Lett. 81, 5150 (2002).

${ }^{19}$ K. Yamaguchi, T. Kaizu, K. Yujobo, and Y. Saito, J. Cryst. Growth 237-239, 1301 (2002).

${ }^{20}$ A. Tackeuchi, Y. Nakata, S. Muto, Y. Sugiyama, T. Usuki, Y. Nishikawa, N. Yokoyama, and O. Wada, Jpn. J. Appl. Phys. 34, L1439 (1995).

${ }^{21}$ Y. I. Mazur, J. W. Tomm, V. Petrov, G. G. Tarasov, H. Kissel, C. Walther, Z. Y. Zhuchenko, and W. T. Masselink, Appl. Phys. Lett. 78, 3214 (2001).

${ }^{22}$ Z. Ma and K. Pierz, Surf. Sci. 511, 57 (2002).

${ }^{23}$ D. F. Cesar, M. D. Teodoro, V. Lopez-Richard, G. E. Marques, E. Marega, Jr., V. G. Dorogan, Y. I. Mazur, and G. J. Salamo, Phys. Rev. B 83, 195307 (2011).

${ }^{24}$ M. Reischle, G. J. Beirne, R. Roßbach, M. Jetter, H. Schweizer, and P. Michler, Phys. Rev. B 76, 085338 (2007).

${ }^{25}$ V. K. Kalevich, M. Paillard, K. V. Kavokin, X. Marie, A. R. Kovsh, T. Amand, A. E. Zhukov, Y. G. Musikhin, V. M. Ustinov, E. Vanelle, and B. P. Zakharchenya, Phys. Rev. B 64, 045309 (2001).

${ }^{26}$ C. A. Duarte, E. C. F. da Silva, A. A. Quivy, M. J. da Silva, S. Martini, J. R. Leite, E. A. Meneses, and E. Lauretto, J. Appl. Phys. 93, 6279 (2003). 